\title{
Novel tetrahydropyrimidinium / palladium system as a convenient catalyst: Suzuki coupling reactions of aryl chlorides
}

\author{
Ismail Özdemir, ${ }^{a, "}$ Serpil Demir, ${ }^{a}$ and Bekir Çetinkaya ${ }^{b}$ \\ ${ }^{a}$ Inönü University,Faculty Science and Arts, Chemistry Department, 44280 Malatya, Turkey \\ ${ }^{b}$ Department of Chemistry, Ege University, 35100 Bornova-Izmir, Turkey \\ E-mail: iozdemir@inonu.edu.tr
}

\begin{abstract}
Four bis(3,4,5,6-tetrahydropyrimidinium) chlorides (2a-d) have been prepared and characterized by conventional spectroscopic methods and elemental analyses. A highly effective, easy to handle and environmentally benign process for palladium mediated Suzuki cross-coupling was developed. The in situ prepared three-component system $\operatorname{Pd}(\mathrm{OAc})_{2} /$ bis(3,4,5,6tetrahydropyrimidinium) chlorides (2a-d) and $\mathrm{K}_{2} \mathrm{CO}_{3}$ catalyses quantitatively the Suzuki crosscoupling of deactivated aryl chlorides.
\end{abstract}

Keywords : $N$-Heterocyclic carbene, tetrahydropyrimidinium, Suzuki, palladium, aryl chlorides, phenylboronic acid

\section{Introduction}

Reactions in which carbon-carbon bond formation took place are important in processes such as bioactive molecule synthesis, drugs and agrochemical syntheis. In particular, a metal catalyst offers a novel model for preparation of such molecules, quite large numbers of papers which predict spectroscopic and structural properties of transition metal complexes have appeared. In general, Suzuki cross-coupling reaction is interpreted as arising from the combination of organoboron compounds with electrophiles catalyzed by palladium complexes. ${ }^{1,2}$

The $\mathrm{C}-\mathrm{Cl}$ bond in chloroarenes is mainly used since chloroarenes are readily available inexpensive materials from an industrial point of view. Traditionally, however, the $\mathrm{C}-\mathrm{Cl}$ bond in chloroarenes has been found to be comparatively inert. ${ }^{3}$ For a along time, the use of unactivated aryl chlorides as substrates in Suzuki-Miyaura cross-coupling was not feasible. Instead, more costly and less readily available aryl iodides and bromides were used. Buchwald ${ }^{4}$ and $\mathrm{Fu}^{5}$ were the first who independently developed catalyst systems based on electron-rich, sterically demanding phosphine ligands, which allow the Suzuki-Miyaura cross-coupling of many unactivated aryl chlorides. However, the major drawback of these is that the phosphine ligands 
are comparatively difficult to prepare or rather expensive. Furthermore, tertiary phosphines require air-free handling to prevent their oxidation and are susceptible to $\mathrm{P}-\mathrm{C}$ bond cleavage at elevated temperatures. ${ }^{6}$

Transition metal complexes incorporating 1,3-diorganyl N-heterocyclic carbene (NHC) ligands, such as imidazol-2-ylidene, imidazolidin-2-ylidene, benzimidazol-2-ylidene and 3,4,5,6-tetrahydropyrimidin-2-ylidene have attracted a great deal of interest in recent years. ${ }^{7-14}$ They are often synthesised via reaction of an azol(in)ium salt (LHX) with a basic salt such as $\mathrm{Pd}(\mathrm{OAc})_{2}$ to give $\mathrm{M}(\mathrm{NHC}) \mathrm{L}_{\mathrm{m}}$.

Research in this area was principally motivated by the use of these complexes as catalyst precursors. Many different catalytic applications of NHC complexes have been described. ${ }^{15-17}$ Palladium-catalysed cross-coupling reactions are particularly attractive because of their versatility for formation of C-C bonds. ${ }^{18-20}$ Sterically hindered, electron-rich alkyl phosphines ${ }^{21}$ and carbene ${ }^{22}$ ligands have received growing interest in recent years. Yet, the phosphine ligands and the phosphine-palladium complexes are labile to air and moisture at high temperatures, placing significant limits on their synthetic utility. Therefore, from a practical point of view, the development of more stable ligands leading to more reactive catalysts have gained importance for palladium-catalyzed Heck and Suzuki coupling reactions. Recently, nucleophilic $N$ heterocyclic carbenes $\left(\mathrm{NHC}^{\prime}\right)^{23}$ with stronger $\sigma$-donor properties than the bulky tertiary phosphines $^{24}$, have emerged as a new family of ligands. In contrast to metal phosphine complexes, the metal-NHC complexes appear to be extraordinarily stable towards heat, air and moisture due to the high dissociation energies of the metal-carbon bond. ${ }^{25}$

The development of new ligands or the application of existing ligands in these reactions, particularly those involving aryl chlorides as substrates, is still of considerable importance. Recently, we have improved procedures Heck and Suzuki reactions of aryl chlorides making use of novel ligands 1,3-bis(dialkyl)imidazolium and benzimidazolinium salts. ${ }^{26}$ Although the nature of the NHC ligand on complexes have tremendous influence on the rate of catalyzed reactions, the use of 3,4,5,6-tetrahydropyrimidinium ligands in coupling reactions is a neglected area. In order to find more efficient palladium catalysts we have prepared a series of new bis $(3,4,5,6$ tetrahydropyrimidinium) chlorides (2) (Scheme 1). We now report on the use of the in situ generated catalytic system composed of commercially available and stable reagents, $\mathrm{Pd}(\mathrm{OAc})_{2}$ as palladium source, bis(3,4,5,6-tetrahydro- pyrimidinium) chlorides (2a-d) as a carbene precursor and $\mathrm{K}_{2} \mathrm{CO}_{3}$ as a base for cross coupling of aryl chlorides with phenylboronic acid.

\section{Results and Discussion}

Bis(3,4,5,6-tetrahydropyrimidinium) chlorides (2a-d) are conventional NHC precursors. According to Scheme 1, the salts (2a-d) were obtained in almost quantitative yield by quarternization of 1 -methyl(3,4,5,6-tetrahydropyrimidine $)^{27}$ in DMF with bis(chloromethyl) benzenes $^{26}$. 


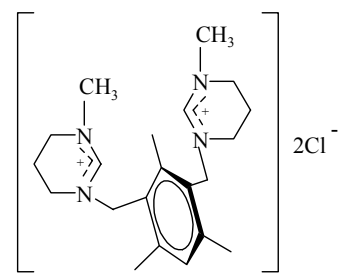

2d
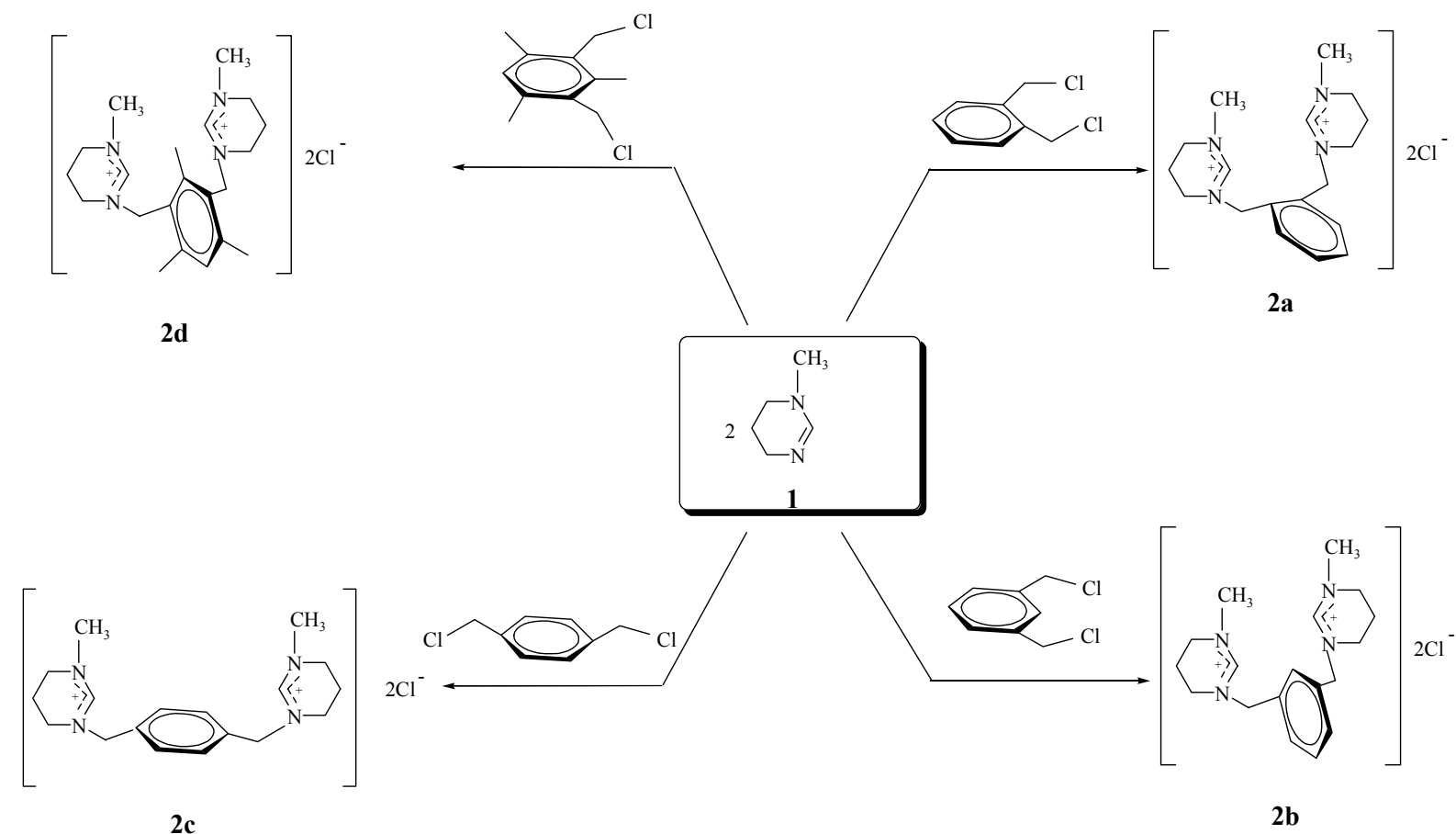

Scheme 1. Synthesis of carbene precursors.

The structures of $\mathbf{2}$ were determined by their characteristic spectroscopic data and elemental analyses. ${ }^{13} \mathrm{C}$ NMR chemical shifts were consistent with the proposed structure; the imino carbon appeared as a typical singlet in the ${ }^{1} \mathrm{H}$-decoupled mode at 154.1, 162.5, 153.9 and 162.4 ppm respectively for pyrimidinium salts $\mathbf{2 a - d}$. The ${ }^{1} \mathrm{H}$ NMR spectra of the pyrimidinium salts further supported the assigned structures; the resonances for $\mathrm{C}(2)-\mathrm{H}$ were observed as sharp singlets at 8.54, 9.79, 9.13 and $9.70 \mathrm{ppm}$ respectively for $\mathbf{2 a - d .}$

The palladium-catalyzed cross-coupling of arylboronic acids with aryl halides has been shown to proceed under a variety of conditions. A wide range of bases and solvents, as well as catalysts, have been employed with varying degrees of success according to the substrates ${ }^{28}$. Here, various bis(3,4,5,6-tetrahydropyrimidinium) salts (2a-d) were compared as ligand precursors under the same reaction conditions. To survey the reaction parameters for the catalytic Suzuki reaction, $\mathrm{Cs}_{2} \mathrm{CO}_{3}, \mathrm{~K}_{2} \mathrm{CO}_{3}$, and $\mathrm{K}_{3} \mathrm{PO}_{4}$ as base and $\mathrm{DMF} / \mathrm{H}_{2} \mathrm{O}(1: 1)$ and dioxane as solvent were used. We found that the reactions performed in $\mathrm{DMF} / \mathrm{H}_{2} \mathrm{O}(1: 1)$ with $\mathrm{Cs}_{2} \mathrm{CO}_{3}$ or $\mathrm{K}_{2} \mathrm{CO}_{3}$ as the base at $80{ }^{\circ} \mathrm{C}$ appeared to be best. We started our investigation with the coupling of $p$ chloroacetophenone and phenylboronic acid, in the presence of $\mathrm{Pd}(\mathrm{OAc})_{2} /$ carbene precursors (2a-d). The results obtained in the presence of 2a-d are summarized (Table 1, entries 1-4). It is evident that the NHC precursors that contain pyrimidinium (2a-d) are the most effective of the examined salts. 
Table 1. The Suzuki coupling reaction of aryl chlorides with phenylboronic acid

\begin{tabular}{cccc}
\hline & & & \\
\hline
\end{tabular}

${ }^{a}$ Reaction conditions : $1.0 \mathrm{mmol}$ of $\mathrm{R}_{-} \mathrm{C}_{6} \mathrm{H}_{4} \mathrm{Cl}-p, 1.5 \mathrm{mmol}$ of phenylboronic acid, $2 \mathrm{mmol}$ $\mathrm{K}_{2} \mathrm{CO}_{3}, 1.50 \mathrm{mmol} \% \mathrm{Pd}(\mathrm{OAc})_{2}, 1.50 \mathrm{mmol} \% 2$, DMF- $\mathrm{H}_{2} \mathrm{O}(3 / 3 \mathrm{~mL}),{ }^{\mathrm{b}}$ purity of compounds is checked by NMR and yields are based on aryl chloride. ${ }^{\mathrm{c}}$ All reactions were monitered by TLC, ${ }^{\mathrm{d}}$ temperature $80^{\circ} \mathrm{C}, 5 \mathrm{~h}$.

Under those conditions, $p$-chloroacetophenone, $p$-chlorotoluene, $p$-chloroanisole and $p$ chlorobenzaldehyde, react very cleanly with phenylboronic acid in goods yields (Table 1, Entries $4,8,12$ and 16). Our results from the screening of four pyrimidinium salts, for Suzuki crosscoupling reaction are summarized in Table 1.

\section{Conclusions}

In conclusion, we have developed a new type of easily prepared bis(3,4,5,6tetrahydropyrimidinium) salts (2a-d) useful as ligands for the Suzuki-Miyaura coupling reaction. Through the use of 2 and $\mathrm{Pd}(\mathrm{OAc})_{2}$ as a pre-catalyst mixture, aryl halides undergo efficient coupling reactions with $\mathrm{C}_{6} \mathrm{H}_{5} \mathrm{~B}(\mathrm{OH})_{2}$ in the presence of $\mathrm{K}_{2} \mathrm{CO}_{3}$. Several trends are readily apparent. First, the procedure is simple and does not require induction periods. Second, the scope 
of this reaction is broad and includes aryl chlorides that are activated or deactivated. Third, all ligands led to good conversions at low catalyst concentration $(1.5 \mathrm{mmol} \%)$. The procedure is simple and efficient towards various aryl halides and does not require induction periods.

\section{Experimental Section}

General Procedures. All reactions for the preparation of 1-methyl-3,4,5,6-tetrahydropyrimidine (1) and bis(1-methyl-3,4,5,6-tetrahydropyrimidine) chlorides ${ }^{27}$ (2a-d) were carried out under argon using standard Schlenk-type flasks. Test reactions for the catalytic activity of catalysts in the Suzuki cross-coupling reactions were carried out in air. All reagents were purchased from the Aldrich Chemical Co. The solvents, were distilled prior to use: $\mathrm{Et}_{2} \mathrm{O}$ over $\mathrm{Na}, \mathrm{DMF}$ over $\mathrm{BaO}$, EtOH over Mg.

All ${ }^{1} \mathrm{H}$ and ${ }^{13} \mathrm{C}-\mathrm{NMR}$ were registered in DMSO- $\mathrm{d}_{6} \cdot{ }^{1} \mathrm{H}$ NMR and ${ }^{13} \mathrm{C}$ NMR spectra were recorded using a Bruker AC300P FT spectrometer operating at $300.13 \mathrm{MHz}\left({ }^{1} \mathrm{H}\right), 75.47 \mathrm{MHz}$ $\left({ }^{13} \mathrm{C}\right)$. Chemical shifts $(\delta)$ are given in ppm relative to TMS and coupling constants $(J)$ in $\mathrm{Hz}$. Infrared spectra were recorded as $\mathrm{KBr}$ pellets in the range $400-4000 \mathrm{~cm}^{-1}$ on a ATI UNICAM 1000 spectrometer. Melting points were measured in open capillary tubes with an Electrothermal-9200 melting point apparatus and are uncorrected. Elemental analyses were performed by TUBITAK (Ankara, Turkey) Microlab.

\section{Preparation of 3,3'-dimethyl-1,1'(1,2-bismethylenebenzene)(3,4,5,6-} tetrahydropyrimidinium) chloride, (2a). To a solution of 1-methyl-3,4,5,6-tetrahydro pyrimidine $(0.98 \mathrm{~g}, 10 \mathrm{mmol})$ in DMF $(10 \mathrm{~mL})$ 1,2-bis(chloromethyl)benzene (0.87 g, $5 \mathrm{mmol})$ was added slowly and the resulting mixture was stirred at room temperature for $5 \mathrm{~h}$. Diethyl ether $(10 \mathrm{~mL})$ was added to obtain a white crystalline solid which was filtered off. The solid was washed with diethylether $(3 \times 10 \mathrm{~mL})$ dried under vacuum and the crude product was recrystallized from ethanol/diethylether. $\mathrm{mp}=150-151{ }^{\circ} \mathrm{C}$, and the yield was $1.65 \mathrm{~g}, 89 \%, v_{(\mathrm{CN})}$ $=1511 \mathrm{~cm}^{-1}$. Anal. Cal. For $\mathrm{C}_{18} \mathrm{H}_{28} \mathrm{~N}_{4} \mathrm{Cl}_{2} ; \mathrm{C}: 58.22, \mathrm{H}: 7.60, \mathrm{~N}: 15.09$; found $\mathrm{C}: 58.25, \mathrm{H}: 7.56$, $\mathrm{N}: 15.10 .{ }^{1} \mathrm{H}$ NMR $\left(\delta, \mathrm{DMSO}_{6}\right): 2.13\left(\mathrm{q}, J=6,0 \mathrm{~Hz}, 4 \mathrm{H}, \mathrm{NCH}_{2} \mathrm{CH}_{2} \mathrm{CH}_{2} \mathrm{~N}\right) ; 3.29$ and $3.48(\mathrm{t}, J$ $\left.=5,6 \mathrm{~Hz}, 8 \mathrm{H}, \mathrm{NCH}_{2} \mathrm{CH}_{2} \mathrm{CH}_{2} \mathrm{~N}\right) ; 3.33\left(\mathrm{~s}, 6 \mathrm{H}, \mathrm{NCH}_{3}\right) ; 4.88\left(\mathrm{~s}, 4 \mathrm{H}, \mathrm{CH}_{2} \mathrm{C}_{6} \mathrm{H}_{4} \mathrm{CH}_{2}\right) ; 7.46(\mathrm{~m}, 4 \mathrm{H}$, $\left.\mathrm{CH}_{2} \mathrm{C}_{6} \mathrm{H}_{4} \mathrm{CH}_{2}\right) ; 8.54(\mathrm{~s}, 2 \mathrm{H}, \mathrm{NCHN}) ;{ }^{13} \mathrm{C}\left\{{ }^{1} \mathrm{H}\right\} \mathrm{NMR}\left(\delta, \mathrm{DMSO}-\mathrm{d}_{6}\right) ; 41.5\left(\mathrm{NCH}_{2} \mathrm{CH}_{2} \mathrm{CH}_{2} \mathrm{~N}\right) ; 42.3$ and $44.9\left(\mathrm{NCH}_{2} \mathrm{CH}_{2} \mathrm{CH}_{2} \mathrm{~N}\right) ; 18.7\left(\mathrm{NCH}_{3}\right) ; 54.9\left(\mathrm{CH}_{2} \mathrm{C}_{6} \mathrm{H}_{4} \mathrm{CH}_{2}\right) ; 132.6,129.4$ and 129.3 $\left(\mathrm{CH}_{2} \mathrm{C}_{6} \mathrm{H}_{4} \mathrm{CH}_{2}\right) ; 154.1(\mathrm{NCHN})$.

Preparation of 3,3'-dimethyl-1,1'(1,3-bismethylenebenzene)(3,4,5,6-tetrahydro pyrimidinium) chloride, (2b). Compound $\mathbf{2 b}$ was prepared in the same way as $\mathbf{2 a}$ from 1methyl-3,4,5,6-tetrahydropyrimidine $(0.98 \mathrm{~g}, 10 \mathrm{mmol})$ in DMF (10 $\mathrm{mL}) \quad 1,3-$ Bis(chloromethyl)benzene $(0.87 \mathrm{~g}, 5 \mathrm{mmol})$ was added slowly to give white crystals of $\mathbf{2} \mathbf{b}$. $\mathrm{mp}=117-118{ }^{\circ} \mathrm{C}$, and the yield was $1.57 \mathrm{~g}, 85 \%, v_{(\mathrm{CN})}=1509 \mathrm{~cm}^{-1}$. Anal. Cal. For $\mathrm{C}_{18} \mathrm{H}_{28} \mathrm{~N}_{4} \mathrm{Cl}_{2}$; C: 58.22, H: 7.60, N: 15.09; found $\mathrm{C}: 58.20, \mathrm{H}: 7.63, \mathrm{~N}: 15.11 .{ }^{1} \mathrm{H}$ NMR 


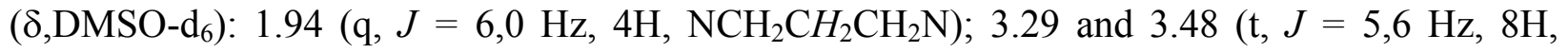
$\left.\mathrm{NCH}_{2} \mathrm{CH}_{2} \mathrm{CH}_{2} \mathrm{~N}\right) ; 3.26$ (s, $\left.6 \mathrm{H}, \mathrm{NCH}_{3}\right) ; 4.66$ (s, $\left.4 \mathrm{H}, \mathrm{CH}_{2} \mathrm{C}_{6} \mathrm{H}_{4} \mathrm{CH}_{2}\right) ; 7.73$ (s, $1 \mathrm{H}, \mathrm{CH}_{2} \mathrm{C}_{6} \mathrm{H}_{4} \mathrm{CH}_{2}$ ); $7.14\left(\mathrm{~m}, \quad 3 \mathrm{H}, \quad \mathrm{CH}_{2} \mathrm{C}_{6} H_{4} \mathrm{CH}_{2}\right) ; 9.79(\mathrm{~s}, \quad 2 \mathrm{H}, \quad \mathrm{NCHN}) ;{ }^{13} \mathrm{C}\left\{{ }^{1} \mathrm{H}\right\} \mathrm{NMR} \quad\left(\delta, \mathrm{DMSO}_{6} \mathrm{~d}_{6}\right): 42.2$ $\left(\mathrm{NCH}_{2} \mathrm{CH}_{2} \mathrm{CH}_{2} \mathrm{~N}\right) ; 42.3$ and $44.9\left(\mathrm{NCH}_{2} \mathrm{CH}_{2} \mathrm{CH}_{2} \mathrm{~N}\right) ; 18.9\left(\mathrm{NCH}_{3}\right) ; 57.8\left(\mathrm{CH}_{2} \mathrm{C}_{6} \mathrm{H}_{4} \mathrm{CH}_{2}\right) ; 154.6$, 134.8, 129.4, 129.1 and $128.5\left(\mathrm{CH}_{2} \mathrm{C}_{6} \mathrm{H}_{4} \mathrm{CH}_{2}\right) ; 162.5(\mathrm{NCHN})$.

Preparation of 3,3'-dimethyl-1,1'(1,4-bismethylenebenzene)(3,4,5,6-tetrahydropyrimidinium) chloride, (2c). Compound $2 \mathrm{c}$ was prepared in the same way as $2 \mathrm{a}$ from 1methyl-3,4,5,6-tetrahydropyrimidine $(0.98 \mathrm{~g}, \quad 10 \mathrm{mmol}) \quad$ in $\mathrm{DMF} \quad\left(\begin{array}{lll}10 & \mathrm{~mL}\end{array}\right) \quad$ 1,4bis(chloromethyl)benzene $(0.87 \mathrm{~g}, 5 \mathrm{mmol})$ was added slowly to give white crystals of $\mathbf{2 c}$. $\mathrm{mp}=282-283{ }^{\circ} \mathrm{C}$, and the yield was $1.72 \mathrm{~g}, 93 \%, v_{(\mathrm{CN})}=1512 \mathrm{~cm}^{-1}$. Anal. Cal. For $\mathrm{C}_{18} \mathrm{H}_{28} \mathrm{~N}_{4} \mathrm{Cl}_{2}$; C: 58.22, H: 7.60, N: 15.09; found C: 58.19, H: 7.61, N: 15.08. ${ }^{1} \mathrm{H}$ NMR $\left(\delta, \mathrm{DMSO}^{-} \mathrm{d}_{6}\right): 1.92(\mathrm{q}$, $\left.J=5.1 \mathrm{~Hz}, 4 \mathrm{H}, \mathrm{NCH}_{2} \mathrm{CH}_{2} \mathrm{CH}_{2} \mathrm{~N}\right) ; 3.33$ and $3.16\left(\mathrm{t}, J=5,1 \mathrm{~Hz}, 8 \mathrm{H}, \mathrm{NCH}_{2} \mathrm{CH}_{2} \mathrm{CH}_{2} \mathrm{~N}\right) ; 3.21(\mathrm{~s}$, $\left.6 \mathrm{H}, \mathrm{NCH}_{3}\right) ; 4.71\left(\mathrm{~s}, 4 \mathrm{H}, \mathrm{CH}_{2} \mathrm{C}_{6} \mathrm{H}_{4} \mathrm{CH}_{2}\right) ; 7.45\left(\mathrm{~s}, 4 \mathrm{H}, \mathrm{CH}_{2} \mathrm{C}_{6} H_{4} \mathrm{CH}_{2}\right) ; 9.13(\mathrm{~s}, 2 \mathrm{H}, \mathrm{NCHN}$ ); ${ }^{13} \mathrm{C}\left\{{ }^{1} \mathrm{H}\right\} \mathrm{NMR}\left(\delta\right.$,DMSO-d $\left.\mathrm{d}_{6}\right): 41.8\left(\mathrm{NCH}_{2} \mathrm{CH}_{2} \mathrm{CH}_{2} \mathrm{~N}\right) ; 41.9$ and $44.5\left(\mathrm{NCH}_{2} \mathrm{CH}_{2} \mathrm{CH} \mathrm{H}_{2} \mathrm{~N}\right) ; 18.7$ $\left(\mathrm{NCH}_{3}\right) ; 56.9\left(\mathrm{CH}_{2} \mathrm{C}_{6} \mathrm{H}_{4} \mathrm{CH}_{2}\right) ; 135.2,129.7,129.2$ and $129.0\left(\mathrm{CH}_{2} \mathrm{C}_{6} \mathrm{H}_{4} \mathrm{CH}_{2}\right) ; 153.9(\mathrm{NCHN})$.

Preparation of 3,3'-dimethyl-1,1'(2,4-bismethylenemesitylene)(3,4,5,6-tetrahydro pyrimidinium) chloride, (2d). Compound 2d was prepared in the same way as 2a from 1methyl-3,4,5,6-tetrahydropyrimidine $(0.98 \mathrm{~g}, \quad 10 \mathrm{mmol})$ in DMF $(10 \mathrm{~mL}) \quad 2,4-$ Bis(chloromethyl)mesitylene (1.08 g, $5 \mathrm{mmol}$ ) was added slowly to give white crystals of $\mathbf{2 d}$. $\mathrm{mp}=264-265{ }^{\circ} \mathrm{C}$, and the yield was $1.86 \mathrm{~g}, 90 \%, v_{(\mathrm{CN})}=1508 \mathrm{~cm}^{-1}$. Anal. Cal. For $\mathrm{C}_{21} \mathrm{H}_{34} \mathrm{~N}_{4} \mathrm{Cl}_{2}$; C: 61.01, H: 8.29, N: 13.55; found C: 61.04, H: 8.27, N: 13.54. ${ }^{1} \mathrm{H}$ NMR (8,DMSO-d $\left.{ }_{6}\right): 2.04$ (q, $\left.J=4.8 \mathrm{~Hz}, 4 \mathrm{H}, \mathrm{NCH}_{2} \mathrm{CH}_{2} \mathrm{CH}_{2} \mathrm{~N}\right) ; 3.13$ and $3.46\left(\mathrm{t}, J=5,1 \mathrm{~Hz}, 8 \mathrm{H}, \mathrm{NCH}_{2} \mathrm{CH}_{2} \mathrm{CH}_{2} \mathrm{~N}\right) ; 3.37$ (s, $\left.6 \mathrm{H}, \mathrm{NCH}_{3}\right) ; 4.94$ (s, 4H, $\left.\left.\mathrm{CH}_{2} \mathrm{C}_{6} \mathrm{H}\left(\mathrm{CH}_{3}\right)_{3}-1,3,5\right) \mathrm{CH}_{2}\right) ; 6.88$ (s, $\left.1 \mathrm{H}, \mathrm{CH}_{2} \mathrm{C}_{6} H\left(\mathrm{CH}_{3}\right)_{3}-1,3,5\right) \mathrm{CH}_{2}$ );

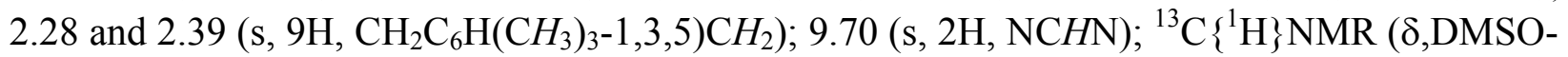
$\left.\mathrm{d}_{6}\right): 41.0 \quad\left(\mathrm{NCH}_{2} \mathrm{CH}_{2} \mathrm{CH}_{2} \mathrm{~N}\right) ; \quad 42.2$ and $45.1 \quad\left(\mathrm{NCH}_{2} \mathrm{CH}_{2} \mathrm{CH}_{2} \mathrm{~N}\right) ; 18.4 \quad\left(\mathrm{NCH}_{3}\right) ; \quad 52.3$ $\left.\left(\mathrm{CH}_{2} \mathrm{C}_{6} \mathrm{H}\left(\mathrm{CH}_{3}\right)_{3}-1,3,5\right) \mathrm{CH}_{2}\right) ; 153.8,139.9,139.0,131.6$ and $\left.127.7\left(\mathrm{CH}_{2} \mathrm{C}_{6} \mathrm{H}\left(\mathrm{CH}_{3}\right)_{3}-1,3,5\right) \mathrm{CH}_{2}\right)$; 20.6 and $\left.16.5\left(\mathrm{CH}_{2} \mathrm{C}_{6} \mathrm{H}\left(\mathrm{CH}_{3}\right)_{3}-1,3,5\right) \mathrm{CH}_{2}\right) ; 162.4(\mathrm{NCHN})$.

\section{General procedure for the Suzuki coupling reaction}

$\mathrm{Pd}(\mathrm{OAc})_{2}(1.5 \mathrm{mmol} \%)$, bis(tetrahydropyrimidinium) chloride, $2(1.5 \mathrm{mmol} \%)$, aryl chloride (1.0 mmol), phenyl boronic acid (1.5 mmol), $\mathrm{K}_{2} \mathrm{CO}_{3}(2 \mathrm{mmol})$, water $(3 \mathrm{~mL})$-DMF $(3 \mathrm{~mL})$ were added to a small Schlenk tube and the mixture was heated at $80{ }^{\circ} \mathrm{C}$ for $5 \mathrm{~h}$. At the conclusion of the reaction, the mixture was cooled, extracted with $\mathrm{Et}_{2} \mathrm{O}$, filtered through a pad of silica gel with copious washing with $\mathrm{Et}_{2} \mathrm{O}$, concentrated and purified by flash chromatography on silica gel. The purity of the compounds was checked by GC and yields are based on aryl chloride, (see Table 1). 


\section{Acknowledgements}

This work was financially supported by the Technological and Scientific Research Council of Turkey TÜBİTAK-CNRS (France) [TBAG-U/181 (106T716)] and Inönü University Research Fund (BAP:2004/Güz8).

\section{References}

1. (a) Miyaura, N.; Suzuki,A. Chem. Rev. 1995, 95, 2457. (b) Suzuki, A. J. Organomet. Chem. 1999, 576, 147. (c) Stanforth, S. P. Tetrahedron 1998, 54, 263. (d) Mingji, D.; Liang, B.; Wang, C.; You, Z.; Xiang, J.; Dong, G.; Chen, J.; Yang, Z. Adv. Synth. Catal. 2004, 346, 1669 .

2. Miyaura, N.; Yanagi,T.; Suzuki, A. Synth. Commun. 1981, 11, 513.

3. Grushin, V. V.; Alper, H. Chem. Rev.1994, 94, 1047.

4. (a) Old, D. W.; Wolfe, J. P.; Buchwald, S. L. J. Am. Chem. Soc.1998, 120, 9722. (b) Wolfe J. P.; Buchwald, S. L. Angew. Chem. Int. Ed. 1999, 38, 2413. (c) Wolfe, J. P.; Singer, R. A.; Yang, B. H.; Buchwald, S. L. J. Am. Chem. Soc. 1999, 121, 9550.

5. (a) Littke, A. F.; Fu, G. C. Angew. Chem. Int. Ed. 1998, 37, 3387. (b) Littke, A. F.; Dai, C.; Fu, G. C. J. Am. Chem. Soc. 2000, 122, 4020. (c) Liu, S.-Y.; Choi, M.J.; Fu, G. C. Chem. Commun. 2001, 2408.

6. Herrmann, W. A. Applied Homogeneous Catalysis with Organometallic Compounds, Cornils, B.; Herrmann, W. A., Eds. VCH: Weinhem, 1996; p 712.

7. Arduengo III, A. J.; Krafcyzk, R. Chem. Unserer Zeit. 1998, 32, 6.

8. Weskamp, T.; Böhm, V. P. W.; Herrmann, W. A. J. Organomet. Chem. 2000, 600, 12.

9. Bourissou, D.; Guerret ,O.; Gabbai, F. P.; Bertrand, G. Chem. Rev. 2000, 100, 39.

10. Magill, A. M.; McGuiness, D. S.; Cavell, K. J.; Britovsek, G. J. P.; Gibson, V. C.; White, J. P.; Williams, D. J.; White, A. H.; Skelton, B. W. J. Organomet. Chem. 2001, 617-618, 546.

11. Crudden, C. M.; Allen, D. P. Coord. Chem. Rev. 2004, 248, 2247.

12. Herrmann, W. A.; Schneider, S. K.; Öfele, K.; Sakamoto, M.; Herdtweck, E. J. Organomet.Chem. 2004, 689, 2441.

13. Mayr, M.; Wurst, K.; Ongania, K. H.; Buchmeiser, M. R. Chem. Eur. J. 2004, 10, 1256.

14. Herrmann W. A. Angew. Chem. Int. Ed. 2002, 41, 1290.

15. Perry, M. C.; Burgess, K. Tetrahedron: Asymm. 2003, 14, 951.

16. Peris, E.; Crabtree, R. H. Coord. Chem. Rev. 2004, 248, 2239.

17. Zapf, A.; Beller, M. Chem. Commun. 2005, 431.

18. Bedford, R. B.; Cazin, C. S. J.; Holder, D. Coord. Chem. Rev. 2004, 248, 2283.

19. Wang, A. E.; Xie, J. H.; Wang, L. X.; Zhou, Q. L. Tetrahedron 2005, 61, 259.

20. Littke, A. F.; Dai, C.; Fu, G. C. J.Am. Chem. Soc. 2000, 122, 4020. 
21. Böhm,V. P. W.; Gstöttmayr, C. W. K.; Weskamp, T.; Herrmann, W. A. J. Organomet. Chem. 2000, 595, 186.

22. Herrmann, W. A.; Weskamp, T.; Böhm, V. P. W. Adv. Organomet. Chem. 2001, 48, 1.

23. Huang, J.; Schanz, H. J.; Stevens, E. D.; Nolan, S. P. Organometallics 1999, 18, 2370.

24. Schwarz, J.; Böhm, V. P. W.; Gardiner, M. G.; Grosche, M.; Herrmann, W. A.; Hieringer, W.; Raudaschl-Sieber, G. Chem. Eur. J. 2000, 6, 1773.

25. Herrmann, W. A.; Öfele, K.; Preysing, D.; Herdtweck, E. J. Organomet. Chem. 2003, 684, 235.

26. (a) Gürbüz, N.; Özdemir, İ.; Demir, S.; Çetinkaya, B. J. Mol. Catal. A:Chem. 2004, 209, 23.

(b) Özdemir, İ.; Gök, Y.; Gürbüz, N.; Yaşar, S.; Çetinkaya, E.; Çetinkaya, B. Polish J. Chem. 2004, 78, 2141. (c) Özdemir, İ.; Gök, Y.; Gürbüz, N.; Çetinkaya, E.; Çetinkaya, B. Synt. Commun. 2004, 34, 4135. (d) Özdemir, İ; Çetinkaya, B.; Demir, S.; Gürbüz, N. Catal. Lett. 2004, 97, 37. (e) Özdemir, İ; Gök, Y.; Gürbüz, N.; Çetinkaya, E.; Çetinkaya, B. Heteroatom Chem. 2004, 15, 419. (f) Özdemir, İ; Alıc1, B.; Gürbüz, N.; Çetinkaya, E.; Çetinkaya, B. J. Mol. Catal. A:Chem. 2004, 217, 37. (g) Gürbüz, N.; Özdemir, İ.; Seçkin, T.; Çetinkaya, B. J. Inorg. and Organomet. Polym. 2004, 14, 149. (h) Özdemir, İ.; Demir, S. Yaşar, S.; Çetinkaya, B. Appl. Organomet. Chem. 2005, 19, 55.

27. (a) Alıcı, B.; Çetinkaya, E.; Çetinkaya, B. Heterocycles 1997, 45, 29. (b) Alıc1, B.; Özdemir, İ.; Gürbüz, N.; Çetinkaya, E.; Çetinkaya, B. Heterocycles 2005, 65, 1439.

28. (a) Bedford, R. B.; Hazelwood, S. L.; Limmert, M.E. Chem. Commun. 2002, 2610. (b) Walker, S. D.; Barder, T. E.; Martinelli, J. R.; Buchwald, S. L. Angew. Chem. Int. Ed. 2004, 43, 1871. (c) Kabakla, G. W.; Wang, L.; Pagni R. M.; Hair, C. M.; Namboodiri, V. Synthesis 2003, 2, 217. (d) Jiang, N.; Ragauskas, A. J. Tetrahedron Lett. 2006, 47, 197. (e) Navarro, O.; Marion, N.; Oonishi, Y.; Kelly III, R. A.; Nolan, S. P. J. Org. Chem. 2006, 71, 685. (f) Schneider, S. K.; Roembke, P.; Julius, G. R.; Loschen, C.; Raubenheimer, H. G.; Frenking, G.; Herrmann, W. A. Eur. J. Inorg. Chem. 2005, 2973. 